\title{
Social response of healthy honeybees towards Nosema ceranae-infected workers: care or kill?
}

\author{
Sarah Biganski ${ }^{1,2}$, Christoph KuRze ${ }^{1,3}$, Matthias Y. Müller ${ }^{1}$, Robin F. A. Moritz ${ }^{1,4,5}$ \\ ${ }^{1}$ Institute for Biology, Martin-Luther-University Halle-Wittenberg, Hoher Weg 4, 06099, Halle (Saale), Germany \\ ${ }^{2}$ Federal Research Centre for Cultivated Plants, Julius Kühn-Institut, Heinrichstraße 243, 64287, Darmstadt, Germany \\ ${ }^{3}$ Center for Infectious Disease Dynamics, Pennsylvania State University, University Park, PA 16802, USA \\ ${ }^{4}$ German Institute for Integrative Biodiversity Research (iDiv), Leipzig, Germany \\ ${ }^{5}$ Department of Zoology and Entomology, University of Pretoria, Pretoria, South Africa
}

Received 14 March 2017 - Revised 21 July 2017 - Accepted 14 November 2017

\begin{abstract}
Honeybees are living densely packed in colonies which extremely facilitates intracolonial pathogen transmission from one individual to another. In addition to the defence by the innate immune system, various behavioural adaptations allow honeybees to avoid or reduce pathogen transmission, also coined 'social immunity'. Here, we show that infections with the intestinal parasite Nosema ceranae lead to altered behaviour going beyond a 'care-kill dichotomy' within the society. We show that the response of healthy workers can be highly variable ranging from avoidance to enhanced social interactions that even lead to killing of infected nestmates in some cases. These behavioural defence mechanisms may help in reducing the spread of the disease in the colony. Thus, honeybees can respond highly variable and not just with one behavioural response after recognising an infected worker even when fighting against the same parasite species.
\end{abstract}

\section{social immunity / behavioural adaptation / honeybee / Nosema / social network}

\section{INTRODUCTION}

Despite numerous advantages of living in large social groups, a major disadvantage is arguably the facilitation of pathogen transmission due to the high density of group members (Cremer et al. 2007; Nunn et al. 2015). This is also true for colonies of the honeybee, Apis mellifera, where often thousands of closely related individuals share a densely packed nest. In addition, the extremely stable and well-controlled nest microclimate provides ideal conditions for rapid pathogen transmission and development (Schmid-Hempel

Electronic supplementary material The online version of this article (https://doi.org/10.1007/s13592-017-0557-8) contains supplementary material, which is available to authorized users.

Corresponding author: S. Biganski, Sarah.Biganski@julius-kuehn.de Manuscript editor: Yves Le Conte
1998; Evans and Schwarz 2011). Interactions amongst nestmates are not just random but often highly directed, including allo-grooming, trophallaxis (food transfer), antennating and contactbased communication, which all promote horizontal pathogen transmission. Once pathogens are inside the colony, they can be rapidly transferred with profound effects on colony health (Loehle 1995; Naug 2008). In particular, intestinal parasites greatly profit from the faecal-oral transmission amongst the adult nestmates such as the globally distributed gut parasite Nosema ceranae (Fries 2010; Higes et al. 2013).

Surprisingly, genome-wide analyses showed that the A. mellifera lacks two thirds of the immune genes that could be identified in the two prime solitary insect models Drosophila melanogaster and Anopheles gambiae (Evans et al. 2006; Barribeau et al. 2015). This apparently decreased capacity of the innate immune response 
is in strong contrast to their colony-based life history. However, honeybees appear to have evolved diverse defence mechanisms against pathogens which act not only on the individual level but also at the social level (Kurze et al. 2016). Social immunity is characterised by a low benefit for the individual bee but an increased fitness advantage for the entire colony (Cremer et al. 2007; Cremer and Sixt 2009; Cotter and Kilner 2010).

There are numerous studies on honeybees addressing the importance of behavioural adaptations to fight infectious diseases. For example, grooming of nestmates and the use of antimicrobial plant substances such as resin for nest construction had been identified as most effective prophylactic traits (Simone-Finstrom and Spivak 2010). Selfmedication as an adaptive therapeutic behaviour in honeybees has been repeatedly suggested as a potential mechanism to reduce infections through antibiotic and antimicrobial compounds in the food (Simone-Finstrom and Spivak 2012; Gherman et al. 2014). Another important and longrecognised trait is hygienic behaviour by which infected brood is removed (Rothenbuhler 1964). Crucial factors of this mechanism are the detection of a pathogen infection by honeybee workers and a change in their behaviour towards the infected brood (Rothenbuhler 1964; Gilliam et al. 1983; Hansen and Brødsgaard 1999). In the light of the excellent capacity of workers to detect infected larvae even in the sealed brood cell (Spivak and Gilliam 1993; Arathi et al. 2000), there seems all reason to expect that they also discriminate amongst infected adults. Indeed, immune-challenged honeybees were shown to express significantly altered cuticular hydrocarbon profiles, which were associated with agonistic behavioural interactions between nestmates (Richard et al. 2008; Richard et al. 2012).

Yet, there are many ways to interact with a diseased nestmate. A worker might offer special care to cure the infected individual. Alternatively, it might however also be adaptive to simply remove infectious workers from the colony. This has been coined as the 'care-kill' dichotomy in colonies of social insects (Schmid-Hempel 1998). Indeed, both might be adaptive strategies to reduce transmission amongst nestmates and the spread of the infection throughout the colony.
Here, we study whether $N$. ceranae-infected honeybee workers are detected by their healthy nestmates and how this may alter their social interactions. To test for potential social responses over the course of the infection, we compare the interactions of one experimentally infected honeybee worker with those of a non-infected control bee in a group of untreated nestmates for 14 days. We quantify not just the interactions of the infected and control bee with their nestmates but also the interactions in the complete social network of groups of workers. We also address the social interactions in groups of bees with variable infection intensities to screen for possible correlations between infection and behavioural response. Both experimental designs were conducted to simulate an initial infection with a single infected honeybee worker in a group of uninfected nestmates and an established infection with more than one Nosema-infected individual.

\section{MATERIAL AND METHODS}

\subsection{Spore purification and specification}

Honeybee foragers were collected at the hive entrances of a colony with a $N$. ceranae infection at the apiary of the Martin-Luther-University Halle-Wittenberg and anaesthetised with carbon dioxide. The alimentary tracts were dissected, pools of five were homogenised in $500 \mu \mathrm{L}$ sterile water and gut content suspensions were checked for spore presence using phase contrast microscopy. Positive suspensions were merged and filtered through filter paper $(10-\mu \mathrm{m}$ pore size). Two repetitive centrifugation steps (8000g for $5 \mathrm{~min}$ ) separated fine debris from spores. Spore purification for following molecular species identification was performed with the triangulation method from Cole (1970). Number of spores was counted using a Fuchs-Rosenthal haemocytometer. Nosema species were verified by standard PCR protocols (Hamiduzzaman et al. 2010; Gisder and Genersch 2013).

\subsection{Experimental inoculation and design}

Frames with capped brood from four colonies were stored in an incubator at $34{ }^{\circ} \mathrm{C}$. Freshly 
emerged uninfected honeybee workers were housed in sterile stainless steel cages with $1 \mathrm{M}$ sucrose solution ad libitum. At the age of 3 days, the bees were individually fed with $2 \mu \mathrm{L}$ sucrose solution containing $10^{5} \mathrm{~N}$. ceranae spores. Uninfected controls were fed with $2 \mu \mathrm{L}$ sucrose solution without Nosema spores. To facilitate the individual feeding procedure and prevent spore transmission via trophallaxis, bees were starved $2 \mathrm{~h}$ prior treatment and individually caged $2 \mathrm{~h}$ afterwards to ensure that spores have passed the honey stomach. All experimental bees were subsequently labelled with numbered Opalith tags glued to their thorax (experiment A: control and infected bee, B: 11 bees, $C$ : 10 bees labelled) and transferred to observation cages in a dark room at $30{ }^{\circ} \mathrm{C}$ and about $60 \%$ r.h.

The bottom of the cages $(10 \times 10 \times 1 \mathrm{~cm})$ was covered by a sheet of honeybee's wax foundation to allow free movement of the bees (Fig. S1, supplementary material). The arena depth of $1 \mathrm{~cm}$ constrained bees turning upside down. An automated infrared (IR) camera system (Basler AG, Germany; resolution $1.4 \mathrm{MP} / 1392 \times 1040$ px, sensor 2/3"; TV zoom lens, EHD imaging 11.5-69 mm, 1:1.4 lens) sequentially recorded eight observation cages to capture bees from the top and contacts between bees on video file continuously recording 11.3 frames per second. The camera was vertically mounted on a 1.5 -m-long sliding rail, $0.5 \mathrm{~m}$ above the observation cages, and was moved by programmed stepper motor (LinearV_HC68, Point Electronics, Halle (Saale), Germany). Two LED-IR spotlights (SAL10, Allround Security, Germany) illuminated the cages when filmed in the dark. The camera moved for cage to cage in 15-min intervals, filming a total of $3 \mathrm{~h}$ homogenously distributed video footage per day. Video analysis was performed with Virtual Dub (vdub32-1.9.1, GNU General Public License, Free Software Foundation Inc., USA).

An interaction between two bees was defined as a contact involving active antennating, trophallaxis or aggressive behaviour (biting, stinging). The number of contacts was counted and interpreted double blind, where the counting person did not know which bees were the infected ones and which were the controls. Furthermore, the survival of all bees was recorded during the experiment. The Nosema spore load per honeybee was quantified at the end of the experiments. We conducted two different experimental designs to test the behavioural interaction tackling two different infection conditions. In a first experimental setup (experiments A and B), one infected honeybee was placed in a group of uninfected workers (A: 49, B: 10 uninfected workers) as it occurs during an initial infection. Healthy workers thus encounter a single infected individual. For this, we monitored the behavioural interactions between the infected honeybee with uninfected controls. In a second approach (experiment C), we studied the behaviour of bees that had experienced different infection intensities to analyse the behavioural interactions in a group of bees with an already established infection.

\subsubsection{Experiment A: contact frequency of target bees and transmission}

One infected honeybee and one control bee were caged together with 48 untreated bees (all bees of same age: 3 days) for 14 days post infection (dpi). If either of them died, this cage was excluded from further observation. For normalisation and equal distribution of contacts over a day, we counted one contact from either uninfected controls or infected bees and subsequently fast-forwarded the video $1 \mathrm{~h}$ to count the next one to obtain independent contacts. In this way, we counted 20 contacts per day for every cage from 1 to $13 \mathrm{dpi}$. This approach of counting a defined number of contacts per day instead of observing a defined lapse of time allows controlling for any changes in overall activity within a group independent of circadian rhythms. We calculated the contact ratio by dividing the number of contacts of the infected bee by the number of contacts of the control bee. Ratios greater than 1 indicate more contacts for the infected bee than for the control bee and vice versa. After 13 dpi, midguts of all honeybee workers were dissected and spores were counted to ascertain the transmission of N. ceranae.

\subsubsection{Experiment B: network analysis}

To obtain a representation of temporal dynamics of the interactions amongst all group members, 
we introduced an infected bee into a group of ten uninfected control bees for 6 dpi in eight cages. Video observations were carried out from 4 to 6 dpi to determine the contacts of all bees per cage. We analysed 72 video sequences, which were evenly spread over the 24-h intervals from 4 to $6 \mathrm{dpi}$. We fast-forwarded the videos for approximately $20 \mathrm{~min}$ (13,560 frames) and observed the first five contacts of each video sequence (in total 360 contacts per cage and day) to determine the releasing and receiving bees. A releasing bee is initiating a contact by starting antennating on the body of a target bee for at least $2 \mathrm{~s}$, whereas receiving bees are target bees of the contact, passively or actively reacting to the contact, i.e. by antennating back. Three different networks per cage were computed by ranking the 11 bees by their number of receiving contacts (indegree network), releasing contacts (outdegree network) and by their number of total contacts (degree network, sum of indegree and outdegree). Increasing ranks indicate a decrease in contact numbers.

\subsubsection{Experiment $C$ : correlational network analysis}

Ten tagged worker bees (age: 3 days) were grouped per observation cage $(N=16)$ and were starved for $1 \mathrm{~h}$ before inoculating them by bulk feeding of $1 \mathrm{~mL}$ sucrose solution containing $5 \times$ $10^{4} N$. ceranae spores $\mu \mathrm{L}^{-1}$ for $2 \mathrm{~h}$ to generate workers with highly variable infection intensities. The dynamics of $N$. ceranae infection in host bees inoculated with $10^{5}$ spores has been described in detail by Forsgren and Fries (2010). Video observations were performed on 1 and 14 dpi. One-hundred independent evenly distributed contacts over both days for each replicate cage were analysed. One contact was defined as a directed touch between two individuals which lasted at least the last $2 \mathrm{~s}$. Indegree and outdegree networks were computed as described above. At the end of the experiment after $14 \mathrm{dpi}, N$. ceranae spore loads of all individual bees were quantified and ranked cage-wise by rank transformation with ties from lowest to highest infection using the individual spore counts. Rank 1 represents bees with the lowest spore count found and rank 8 the highest. Hence, each individual spore count was given an own rank ascending with the number of spores. The aim of this experiment was to examine the contacts of less infected individuals to higher infected ones. Correlations just based on spore counts do not take into account the infection level of an individual in relation to the other group members; i.e. a spore count of 1000 might reflect a highest infection level in one group but the lowest in another where the other group members had higher infection levels. We therefore ranked all individuals according to their infection level within a group. The infection ranks across groups were then used for a subsequent network analyses. If workers in the group had died by day 14 p.i., ranks were scaled according to a lower number of bees in the group. Cages with less than eight survived bees on day 14 p.i. were removed from further analyses. In cages with more than eight survived bees, randomly chosen individuals were removed from further analyses until eight bees were remaining for one cage. The same bees were removed for day 1 p.i. Individual spore counts per cage were assigned to one individual rank. If two or more bees had the same spore count, an interrank was assigned according to the number of bees sharing the same rank.

The average number of contacts was used for correlational analyses of ranked infection levels and the social network parameter indegree and outdegree contacts. Therefore, we calculated the mean contact number for both indegree and outdegree of all individuals from 16 cages that were assigned to the same rank of infection intensity. Following, we compiled a correlation by mean indegree and outdegree contacts depending on the ranked infection levels.

\subsection{Statistical analysis}

\subsubsection{Experiments $A$ and B: contact frequency and network}

The change of the contact frequency over experimental days was analysed by Spearman rank correlation. Differences between the number of contacts of infected and control bee and the indegree and outdegree in the network were analysed with paired $t$ tests by comparing the indegree of control and infected, the outdegree 
of control and infected and outdegree and indegree of infected bee as well as for the control bee. Additionally, $\chi^{2}$ tests assessed pair wise comparisons of indegree and outdegree between the infected bee and all uninfected nestmates in one cage.

\subsubsection{Experiment $C$ : correlational network analysis}

Eight ranks of infection levels were assigned, ranging from low (rank 1) to high (rank 8) infection levels, using Nosema spore count data determined on day 14 post infection of from all individuals of the experiment $C$. Spearman rank correlation was used for linear fitting indegree and outdegree with ranked infection levels. The mean indegree and outdegree values were calculated by grouping individual indegrees and outdegrees of all nestmates with the same rank of infection level. Differences in mean indegrees and outdegrees of all infection ranks were compared on 1 and 14 dpi and tested with Kruskal-Wallis ANOVA and oneway ANOVA, respectively.

All statistical analyses were carried out using the software Statistica 8.0 (StatSoft, Tulsa, OK, USA). Graphs were compiled with $\mathrm{R}$ version 3.3.2 (2016-10-31).

\section{RESULTS}

\subsection{Experiment A: contact frequency of target bees}

All individuals in a cage were screened for Nosema infection after 14 dpi. All uninfected control bees and all untreated individuals were free of Nosema at the end of the experiments. Inoculated bees that survived 13 dpi were infected with $3.9 \pm 0.85 \times 10^{6}$ spores per individual $(N=$ $8)$, whereas bees dying earlier were higher infected with $9.04 \pm 3.14 \times 10^{6}$ spores $(N=9)$. One third $(N=8)$ of the infected bees $(N=24)$ were killed by their healthy nestmates by extreme aggressive behaviour including stinging and dismembering the body parts of the infected workers within 7 dpi. These groups were subsequently removed from contact ratio analysis to evaluate non-aggressive interactions amongst healthy and infected workers. In addition, the killing of the infected bee led to a termination of the experiment in these cages due to its definition as target bee.

The contact frequency of target bees shows an increase of contacts for the infected bees and a decrease of contacts for the control bees, respectively, over the experimental days. The mean contact ratio (infected/control) increased significantly from $1 \mathrm{dpi}$ (mean $\pm \mathrm{SE}=1.04 \pm 0.08)$ to $13 \mathrm{dpi}($ mean \pm $\mathrm{SE}=1.59 \pm 1.06$ ) over time (adjusted $r^{2}=0.585, F$ $(1,11)=15.5, P=0.002)$ (Figure 1).

\subsection{Experiment B: network analysis}

Both the number of total contacts (degree) and the released contacts (outdegree) in the social network did not significantly differ between infected $\left(t_{68}=-1.47, P=0.15\right)$ and control bees $\left(t_{68}=-0.39, P=0.67\right)$. However, the number of received contacts (indegree) was significantly higher in infected bees than the control bees $\left(t_{68}=-2.75, P<0.01\right)$ (Figure 2), confirming the results of the first experiment. Furthermore, the infected bees had significantly more indegree

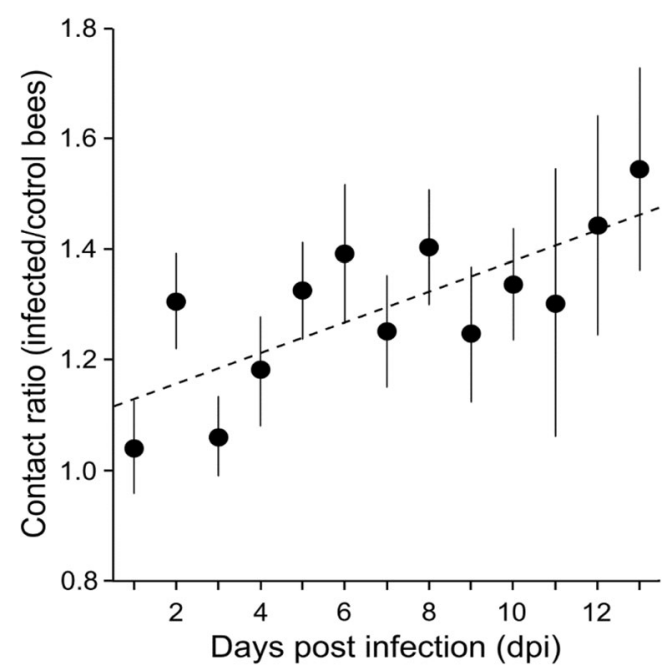

Figure 1. Linear regression of mean $\pm \mathrm{SE}$ contact ratio of the contacts of infected to control bee over $13 \mathrm{dpi}$ with linear fit function $(y=1.09+0.03 \times x)$. The contact ratio of infected to control bees significantly increased from 1 to 13 dpi by linear regression analysis $\left(r^{2}=0.585, F(1,11)=15.5, P=0.002\right)$, whereby contacts of infected bees increased and contacts of control bees decreased over experimental days, respectively. 


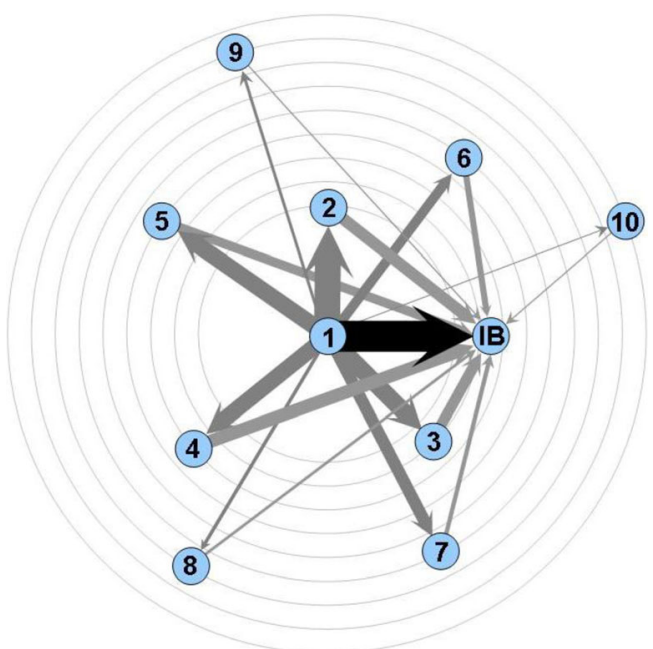

Figure 2. Average degree network for eight groups each containing one infected honeybee (IB) and ten uninfected control bees (1 to 10). The direction of arrows shows which node (= individual bee) is the receiver, and thickness of arrows illustrates the mean degrees to this specific node and between this pair. Distance of arrows and the position in the network (circles) include the mean degree to all other nodes. The central bees (1) of the average degree network release significantly more contacts towards the infected bees (IBs) than to any other bee $\left(\chi^{2}(1, N=71)=6.09\right.$, $P=0.01$ ), but within the network individual (2) has a more central position than (IB) for having more total contacts. Accordingly, the infected bees receive significantly more contacts from the central bees than from any other group member $\left(\chi^{2}(1, N=71)=11.58, P<\right.$ $0.001)$.

contacts than outdegree contacts (paired $t_{7}=2.79$, $P=0.02$ ), whereas there was no significant difference in the controls (paired $t_{61}=-0.75, P=$ $0.45)$. Central bees of the networks released significantly more contacts towards the infected bees than to any other bee $\left(\chi^{2}(1, N=71)=6.09, P=\right.$ $0.01)$. Accordingly, the infected bees receive significantly more contacts from the central bee than from any other group member $\left(\chi^{2}(1, N=71)=\right.$ 11.58, $P<0.001$ ) (Figure 2).

\subsection{Experiment C: correlational network analysis}

Individual spore loads ranged from 0 up to $9 \times$ $10^{7}$ spores per bee after $14 \mathrm{dpi}$, allowing to rank all bees per group from the lowest to the highest infection level.

On day 1 p.i., neither indegree $(r \mathrm{~s}=0.231$, $P=0.428 ; H=13.342, d f=13, P=0.422)$ nor outdegree interactions $(r \mathrm{~s}=-0.174, P=0.553$; $H=18.409, d f=13, P=0.143)$ revealed any significant correlations with the infection levels determined at the end of the experiment (Figure 3a, b).

However, on 14 dpi (Figure 4a), we found a significant negative correlation between the average indegree contacts and the ranked infection levels $(r \mathrm{~s}=-0.736, P=0.003 ; F=1.148, d f=$ $13, P=0.331)$. In contrast, the outdegree contacts (Figure $4 \mathrm{~b}$ ) neither showed a significant difference amongst grouped infection levels $(H=$ 14.304, $d f=13, P=0.353$ ) nor a significant correlation $(r s=-0.07, P=0.814)$.

\section{DISCUSSION}

Microbial and parasitic infections commonly have strong impact on physiological but also on behavioural level of host insects as it was shown in several studies before (Oi and Pereira 1993; Little et al. 2006). We show that infections can profoundly change the social interactions amongst honeybee workers. These changes in behaviour can be initiated either by the infected individual or by healthy nestmates. Because it is often difficult to identify which individual acts as initiator of a certain interaction, we used social networks to qualitatively measure the initiative and responsive behaviour (Otterstatter and Thomson 2007). In this study, we simulated different conditions in a honeybee colony: firstly, an incipient colony infection where one single infected nestmate meets with healthy workers (Schmid-Hempel 1995) and secondly, an established colony infection with variable infection intensities amongst the workers. Our data show that the social responses towards infected individuals do not follow one strict pattern to deal with highly infected individuals that are likely to play an important role in parasite transmission within the colony.

We found that in the initial infection condition, the healthy nestmates seem to be the drivers of the interactions, supporting the results by Richard et al. (2008). Healthy bees regularly aggressively 

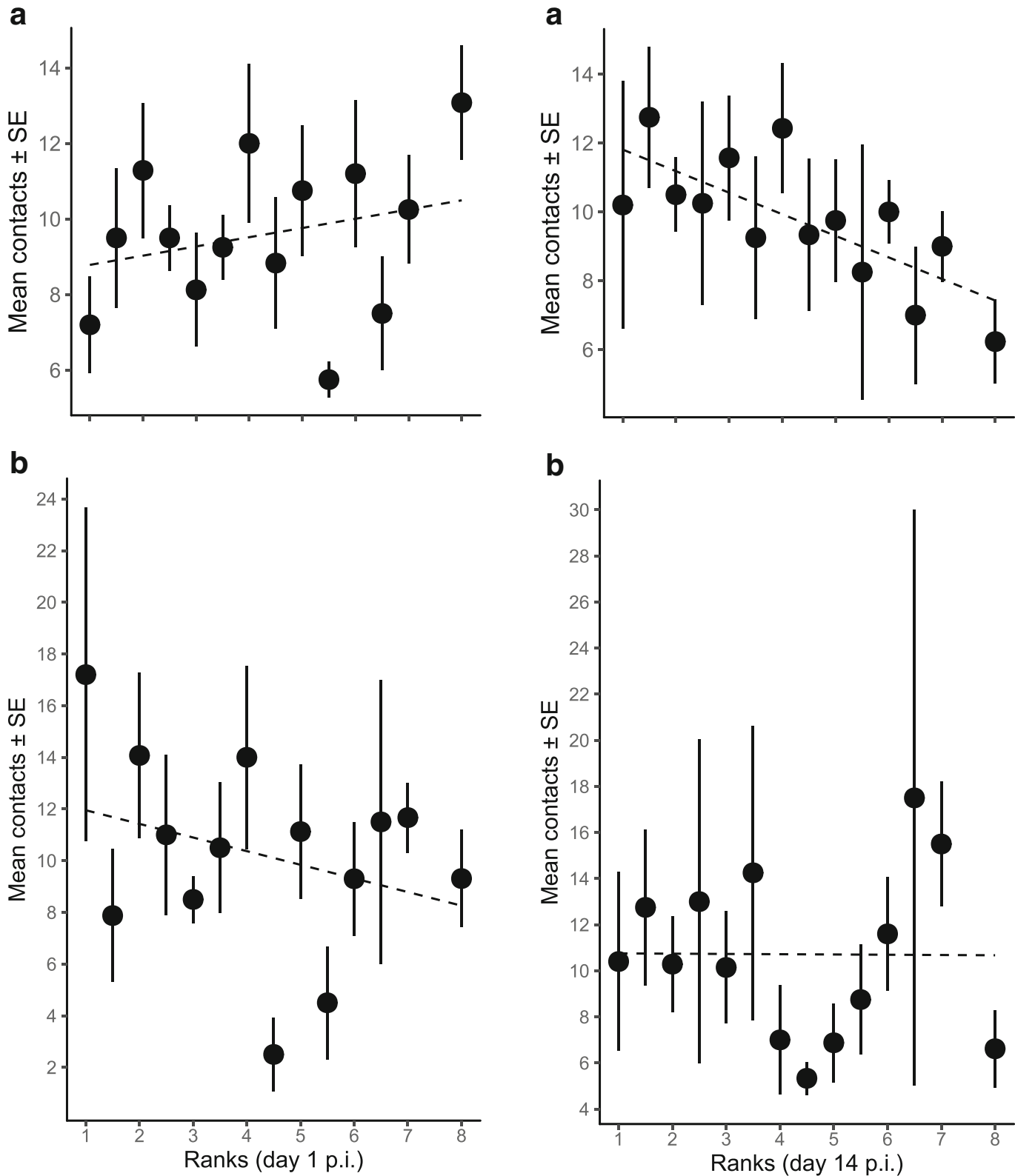

Figure 3. a Mean indegree and $\mathbf{b}$ outdegree contacts \pm SE on 1 dpi grouped by ranked infection levels on day 14 p.i. with linear fit function (a $y=8.731+0.114 \times x$, b $y=12.287-0.276 \times x)$. Neither mean indegree contacts $(r \mathrm{~s}=0.231, P=0.428)$ nor outdegree contacts $(r$ $\mathrm{s}=-0.174, P=0.553)$ show a significant correlation.

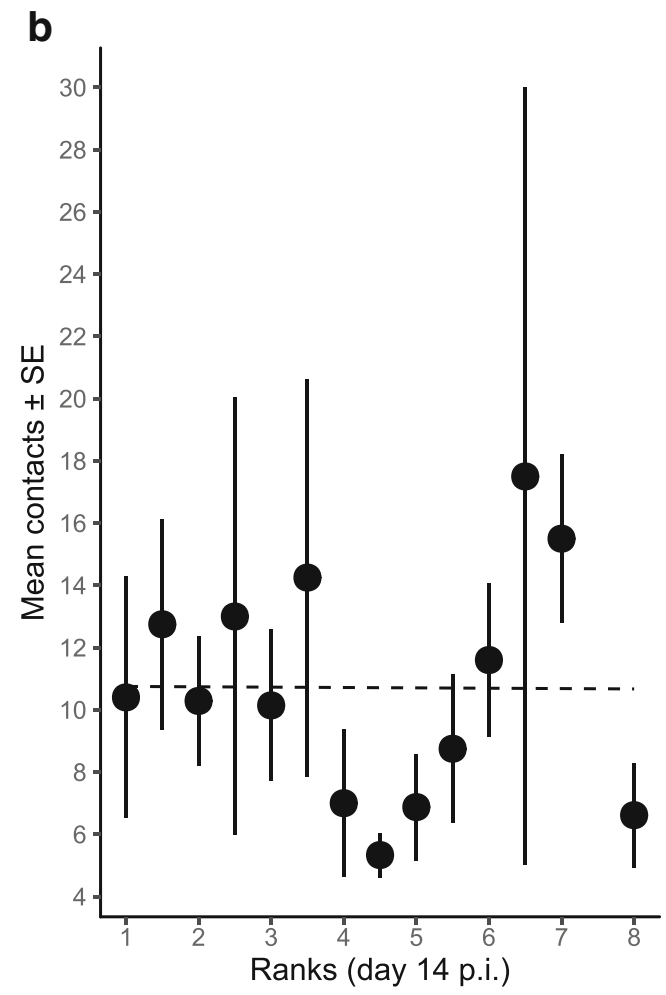

Figure 4. a Mean indegree and $\mathbf{b}$ outdegree contacts \pm SE on 14 dpi grouped by ranked infection levels on day 14 p.i. with linear fit function (a $y=12.120-0.312 \times$ $x$, b $y=10.679+0.008 \times x)$. Mean indegree contacts show a significant negative correlation with increasing infection intensity $(r \mathrm{~s}=-0.736, P=0.003)$ but not outdegree contacts $(r \mathrm{~s}=-0.07, P=0.814)$.

attacked single infected individuals amongst a healthy workers killed infected bees with dismembering the body parts thorax, head and abdomen, 
whereas the control bees remained unmolested. This result dramatically illustrates how honeybee workers can modify their behaviour towards infected nestmates. Indeed, forcing colony members out of the colony is a well-documented behaviour in the honeybee colony (Arathi et al. 2000; Rosengaus and Traniello 2001). If not killed, infected honeybees encountered significantly more contacts than the non-infected control bees within a group of healthy nestmates. This interaction frequency increased significantly during the course of the infection (Figure 1). Such behaviour was already shown for the leaf-cutting ant Acromyrmex echinatior, in which parasitised individuals received more care and were more frequently groomed by healthy nestmates (Hughes et al. 2002; Walker and Hughes 2009). We also found a quantitative increase of receiving contacts by $N$. ceranae -infected bees particularly by the central uninfected bees. Hence, the infected individuals might have received more attendance and care than healthy nestmates which has also been reported in previous behavioural studies (SchmidHempel 1998; Cremer et al. 2007; Bos et al. 2012).

Interestingly, we found an opposite change for the indegree interactions in our second experimental setup, in which all nestmates carried a Nosema infection.

The higher the infection level in a bee is, the more peripheral its position within the indegree networks is. Thus, the contacts with highly diseased bees became reduced rather than enhanced. The health status of the group might therefore be an additional factor influencing the plasticity of behaviour to counter a pathogen thread and vice versa (Evans and Spivak 2010). Low infected bees rather avoided interactions with heavily infected nestmates, which may be a way to reduce the transmission of the pathogen in the colony (Stroeymeyt et al. 2014). It seems that the infection level of a given worker governs the behavioural threshold to interact with an infected fellow worker. The uninfected worker may prefer to either kill or care; the low infected one avoids the highly infected nestmate. Hygienic behaviour of honeybee workers with uncapping and the removal of infected brood is a textbook example (Rothenbuhler 1964) in the context of an infection (Spivak and Gilliam 1993; Spivak and Gilliam
1998). Another case is the Varroa-sensitive hygienic behaviour (Harbo and Harris 2005; Harbo and Harris 2009). Also, here, nest members (in this case: larvae) are killed and removed from the colony. In our experiments, infected workers were killed in similarly extreme way as reported for ants (De Souza et al. 2008). In a colony setting, this would probably not have occurred because the infected workers would have left the colony before (Dussaubat et al. 2013; Goblirsch et al. 2013). Richard et al. (2008) further support this idea of immune response-induced modulations of social interactions in honeybees. The response towards the immune-stimulated honeybees was expressed by significantly more non-agonistic behaviour such as antennating and allo-grooming. In contrast, agonistic behaviour was not significantly increased in comparison to a control group (Richard et al. 2008). The attacks observed by Richard et al. (2008) were described as opening mandibles and biting. Similarly, we observed such aggressive behaviour towards infected individuals in our first study with only one infected honeybee amongst uninfected nestmates. We recorded that uninfected bees were stinging and chasing infected nestmates. This behavioural phenomenon has been coined as the 'care-kill dichotomy' by Paul Schmid-Hempel (1998). However, we also found a third type of behavioural response similar to quarantine in addition to the 'care-kill dichotomy'. Infected workers received less contacts by others as indicated by the reduced indegree although they did not significantly change their outdegree. Hence, the avoidance of infected nestmates might reflect a third defence strategy against $N$. ceranae in the honeybee colony (Kurze et al. 2016). This variability in behavioural responses shows the extremely high plasticity of behaviour in honeybees contributing to the overall social immunity. Murray et al. (2015) found that infected bees are characterised by an altered cuticular hydrocarbon $(\mathrm{cCH})$ profile. Although they could not find specific behavioural changes in their study, we cannot exclude that changes in the $\mathrm{cCH}$ profile are important markers allowing for the behavioural modifications found in our study and in bees infected with deformed wing virus (Baracchi et al. 2012).

In conclusion, it seems clear that the honeybees may respond in a most context-dependent way, and that there is no singular behavioural response 
in dealing with infected workers. We should not be surprised to see very specific responses of honeybee workers towards infected nestmates depending not just on the type of pathogen and infection level of a specifically infected bee but also on the health status of the entire colony.

\section{ACKNOWLEDGEMENTS}

We thank Astrid Mosemann for her support in conducting one of the experiments and data collection. Financial support was granted by Deutsche Forschungsgemeinschaft (DFG; Priority Programme SPP 1399; grant number MO373/26-2).

\section{AUTHOR'S CONTRIBUTION}

SB, CK and RFAM contributed equally to the manuscript. SB and MYM performed the experiments and analyses. All authors read and approved the final manuscript.

\section{COMPLIANCE WITH ETHICAL STANDARDS}

Conflict of interest The authors declare that they have no conflict of interest.

\begin{abstract}
Réponse sociale des abeilles en bonne santé envers les ouvrières infectées par Nosema ceranae: les soigner ou les éliminer?
\end{abstract}

immunité sociale / adaptation comportementale / Apis mellifera / Nosema / réseau social

Die soziale Antwort gesunder Honigbienen gegenüber Nosema ceranae-infizierten Arbeiterinnen: Pflegen oder töten?

Soziale Immunität / adaptatives Verhalten / Honigbiene / Nosema / soziales Netzwerk

\section{REFERENCES}

Arathi, H., Burns, I., Spivak, M. (2000) Ethology of hygienic behaviour in the honey bee Apis mellifera $\mathrm{L}$. (Hymenoptera: Apidae): behavioural repertoire of hygienic bees. Ethology 106 (4):365-379
Baracchi, D., Fadda, A., Turillazzi, S. (2012) Evidence for antiseptic behaviour towards sick adult bees in honey bee colonies. J. Insect Physiol. 58 (12):1589-1596

Barribeau, S.M., Sadd, B.M., du Plessis, L., Brown, M.J.F., Buechel, S.D. et al. (2015) A depauperate immune repertoire precedes evolution of sociality in bees. Genome Biol. 16 (1):83

Bos, N., Lefevre, T., Jensen, A.B., D'ettorre, P. (2012) Sick ants become unsociable. J. Evol. Biol. 25 (2):342-351

Cole, R.J. (1970) The application of the "triangulation" method to the purification of Nosema spores from insect tissues. J. Invertebr. Pathol. 15 (2):193-195

Cotter, S., Kilner, R. (2010) Personal immunity versus social immunity. Behav. Ecol. 21 (4):663-668

Cremer, S., Sixt, M. (2009) Analogies in the evolution of individual and social immunity. Philos. Trans. R. Soc. B Biol. Sci. 364 (1513):129-142

Cremer, S., Armitage, S.A., Schmid-Hempel, P. (2007) Social immunity. Curr. Biol. 17 (16):R693-R702

De Souza, D.J., Van Vlaenderen, J., Moret, Y., Lenoir, A. (2008) Immune response affects ant trophallactic behaviour. J. Insect Physiol. 54 (5):828-832

Dussaubat, C., Maisonnasse, A., Crauser, D., Beslay, D., Costagliola, G., Soubeyrand, S., Kretzchmar, A., Le Conte, Y. (2013) Flight behavior and pheromone changes associated to Nosema ceranae infection of honey bee workers (Apis mellifera) in field conditions. J. Invertebr. Pathol. 113 (1):42-51

Evans, J.D., Schwarz, R.S. (2011) Bees brought to their knees: microbes affecting honey bee health. Trends Microbiol. 19(12):614-620

Evans, J.D., Spivak, M. (2010) Socialized medicine: individual and communal disease barriers in honey bees. J. Invertebr. Pathol. 103:S62-S72

Evans, J.D., Aronstein, K., Chen, Y.P., Hetru, C., Imler, J.L., Jiang, H., Kanost, M., Thompson, G.J., Zou, Z., Hultmark, D. (2006) Immune pathways and defence mechanisms in honey bees Apis mellifera. Insect Mol. Biol. 15(5):645-656

Forsgren, E., Fries, I. (2010) Comparative virulence of Nosema ceranae and Nosema apis in individual European honey bees. Vet. Parasitol. 170 (3):212-217

Fries, I. (2010) Nosema ceranae in European honey bees (Apis mellifera). J. Invertebr. Pathol. 103:S73-S79

Gherman, B.I., Denner, A., Bobiş, O., Dezmirean, D.S., Mărghitaş, L.A., Schlüns, H., Moritz, R.F.A., Erler, S. (2014) Pathogen-associated self-medication behavior in the honeybee Apis mellifera. Behav. Ecol. Sociobiol. $68(11): 1777-1784$

Gilliam, M., Taber III, S., Richardson, G.V. (1983) Hygienic behavior of honey bees in relation to chalkbrood disease. Apidologie 14(1):29-39

Gisder, S., Genersch, E. (2013) Molecular differentiation of Nosema apis and Nosema ceranae based on speciesspecific sequence differences in a protein coding gene. J. Invertebr. Pathol. 113 (1):1-6 
Goblirsch, M., Huang, Z.Y., Spivak, M. (2013) Physiological and behavioral changes in honey bees (Apis mellifera) induced by Nosema ceranae infection. PLoS One 8 (3): e58165

Hamiduzzaman, M.M., Guzman-Novoa, E., Goodwin, P.H. (2010) A multiplex PCR assay to diagnose and quantify Nosema infections in honey bees (Apis mellifera). J. Invertebr. Pathol. 105(2):151-155

Hansen, H., Brødsgaard, C.J. (1999) American foulbrood: a review of its biology, diagnosis and control. Bee World 80 (1):5-23

Harbo, J.R., Harris, J.W. (2005) Suppressed mite reproduction explained by the behaviour of adult bees. J. Apic. Res. $44(1): 21-23$

Harbo, J.R., Harris, J.W. (2009) Responses to Varroa by honey bees with different levels of Varroa sensitive hygiene. J. Apic. Res. 48(3):156-161

Higes, M., Meana, A., Bartolomé, C., Botías, C., MartínHernández, R. (2013) Nosema ceranae (Microsporidia), a controversial 21st century honey bee pathogen. Environ. Microbiol. Rep. 5(1):17-29

Hughes, W.O., Eilenberg, J., Boomsma, J.J. (2002) Tradeoffs in group living: transmission and disease resistance in leaf-cutting ants. Proc. R. Soc. Lond. B Bio. 269 (1502):1811-1819

Kurze, C., Routtu J., Moritz R.F.A. (2016) Parasite resistance and tolerance in honeybees at the individual and social level. Zoology 119(4):290-297

Little, A.E., Murakami, T., Mueller, U.G., Currie, C.R. (2006) Defending against parasites: fungus-growing ants combine specialized behaviours and microbial symbionts to protect their fungus gardens. Biol. Lett. 2(1):12-16

Loehle, C. (1995) Social barriers to pathogen transmission in wild animal populations. Ecology 76(2):326-335

Murray, Z.L., Keyzers, R.A., Barbieri, R.F., Digby, A.P., Lester, P.J. (2015) Two pathogens change cuticular hydrocarbon profiles but neither elicit a social behavioural change in infected honey bees, Apis mellifera (Apidae: Hymenoptera). Aust. J. Entomol. 55 (2):147153

Naug, D. (2008) Structure of the social network and its influence on transmission dynamics in a honeybee colony. Behav. Ecol. Sociobiol. 62 (11):1719-1725

Nunn, C.L., Jordan, F., McCabe, C.M., Verdolin, J.L., Fewell, J.H. (2015) Infectious disease and group size: more than just a numbers game. Philos. Trans. R. Soc. Lond., B, Biol. Sci. 370 (1669):20140111. doi: https://doi.org/10.1098/rstb.2014.0111
Oi, D.H., Pereira, R.M. (1993). Ant behavior and microbial pathogens (Hymenoptera: Formicidae). Fla. Entomol. $76: 63-74$

Otterstatter, M.C., Thomson, J.D. (2007) Contact networks and transmission of an intestinal pathogen in bumble bee (Bombus impatiens) colonies. Oecologia 154 (2):411-421

Richard, F.J., Aubert, A., Grozinger, C. (2008) Modulation of social interactions by immune stimulation in honey bee, Apis mellifera, workers. BMC Biology 6 (1):50

Richard, F.J., Holt, H.L., Grozinger, C.M. (2012) Effects of immunostimulation on social behavior, chemical communication and genome-wide gene expression in honey bee workers (Apis mellifera). BMC Genomics 13 (1):558

Rosengaus, R.B., Traniello, J.F. (2001) Disease susceptibility and the adaptive nature of colony demography in the dampwood termite Zootermopsis angusticollis. Behav. Ecol. Sociobiol. 50 (6):546-556

Rothenbuhler, W.C. (1964) Behavior genetics of nest cleaning in honey bees. IV. Responses of F1 and backcross generations to disease-killed brood. Am. Zool. 4 (2):111-123

Schmid-Hempel, P. (1995) Parasites and social insects. Apidologie 26 :255-271

Schmid-Hempel, P. (1998) Parasites in social insect. Princeton Univ. Press. Princeton

Simone-Finstrom, M., Spivak, M. (2010) Propolis and bee health: the natural history and significance of resin use by honey bees. Apidologie 41 (3):295-311

Simone-Finstrom, M.D., Spivak, M. (2012) Increased resin collection after parasite challenge: a case of selfmedication in honey bees? PLoS One 7 (3):e34601

Spivak, M., Gilliam, M. (1993) Facultative expression of hygienic behaviour of honey bees in relation to disease resistance. J. Apic. Res. 32:147-157

Spivak, M., Gilliam, M. (1998) Hygienic behaviour of honey bees and its application for control of brood diseases and Varroa: Part II. Studies on hygienic behaviour since the Rothenbuhler era. Bee World 79(4):169-186

Stroeymeyt, N., Casillas-Pérez, B., Cremer, S. (2014) Organisational immunity in social insects. Curr. Opin. Insect Sci. $5: 1-15$

Walker, T.N., Hughes, W.O. (2009) Adaptive social immunity in leaf-cutting ants. Biol. Lett. 5 (4):446-448 